\title{
Limb remote ischemic per-conditioning protects heart against ischemia/reperfusion injury through opioid system in rats
}

\begin{tabular}{|c|c|}
\hline Journal: & Canadian Journal of Physiology and Pharmacology \\
\hline Manuscript ID & cjpp-2016-0585.R2 \\
\hline Manuscript Type: & Article \\
\hline Date Submitted by the Author: & 23-Mar-2017 \\
\hline Complete List of Authors: & $\begin{array}{l}\text { Zhang, Li; Department of Physiology, Hebei Medical University; Orthopedic } \\
\text { Department of Third Hospital, Hebei Medical University } \\
\text { Guo, Hui; Department of Gynaecology and Obstetrics, the Fourth Hospital, } \\
\text { Hebei Medical University } \\
\text { Yuan, Fang; Department of Physiology, Hebei Medical University; Hebei } \\
\text { Collaborative Innovation Center for Cardio-cerebrovascular Disease } \\
\text { Hong, Zeng-chao; Operation room of Third Hospital, Hebei Medical } \\
\text { University } \\
\text { Tian, Yan-ming; Department of Physiology, Hebei Medical University } \\
\text { Zhang, Xiang-jian; Hebei Collaborative Innovation Center for Cardio- } \\
\text { cerebrovascular Disease } \\
\text { Zhang, Yi; Department of Physiology, Hebei Medical University; Hebei } \\
\text { Collaborative Innovation Center for Cardio-cerebrovascular Disease }\end{array}$ \\
\hline $\begin{array}{r}\text { Is the invited manuscript for } \\
\text { consideration in a Special } \\
\text { Issue?: }\end{array}$ & N/A \\
\hline Keyword: & $\begin{array}{l}\text { Remote ischemic per-conditioning, cardiac protection, ischemia/ } \\
\text { reperfusion, opioid receptors, ATP-sensitive } \mathrm{K}+\text { channels }\end{array}$ \\
\hline
\end{tabular}


Limb remote ischemia per-conditioning protects heart against ischemia/reperfusion injury through opioid system in rats

Li Zhang ${ }^{1,2^{*}}$, Hui Guo ${ }^{3 *}$, Fang Yuan ${ }^{1,4}$, Zeng-chao Hong ${ }^{5}$, Yan-ming Tian ${ }^{1}$, Xiang-jian Zhang $^{4}$, Yi Zhang ${ }^{1,4^{* *}}$

${ }^{1}$ Department of Physiology, Hebei Medical University, Shijiazhuang, 050017, P.R. China

2 Orthopedic Department of Third Hospital, Hebei Medical University, Shijiazhuang, 050000, P.R. China

3 Department of Gynaecology and Obstetrics, the Fourth Hospital, Hebei Medical University, Shijiazhuang 050000, P.R. China

4 Hebei Collaborative Innovation Center for Cardio-cerebrovascular Disease, Shijiazhuang 050000, P.R. China

5 Operation room of Third Hospital, Hebei Medical University, Shijiazhuang, 050000, P.R. China

Running title: Cardiac protection of per-conditioning

*These authors contribute equally to this study.

** Corresponding author: Professor Yi Zhang, Ph.D., Department of Physiology, Hebei Medical University, Shijiazhuang 050017, P. R. China.

Tel: 86311 86265663, Fax: 86311 86266811, Email: zhyhenryphy@163.com 
Abstract: Remote ischemia per-conditioning (RPerC) has been demonstrated to have cardiac protection, but the underlying mechanism remains unclear. This study aimed to investigate the mechanism underlying cardiac protection of RPerC. Adult male Sprague-Dawley rats were used in this study. Cardiac ischemia/reperfusion (I/R) was induced by 30-min occlusion and 3-h reperfusion of left anterior descending coronary artery. RPerC were performed by 5-min occlusion of right femoral artery followed by 5-min reperfusion for three times during cardiac ischemia. Hemodynamics, left ventricular function, arrhythmia, and infarct area were measured. Protein expression levels of eNOS, iNOS, PKCe, and PKC $\delta$ in myocardium were assayed. During I/R, systolic artery pressure and left ventricular function were decreased, infarct area was increased, and arrhythmia score was increased $(P<0.05)$. However, changes of the above parameters were significantly attenuated in RPerC-treated rats compared with control rats $(P<0.05)$. The cardiac protective effects of RPerC were prevented by naloxone or glibenclamide. Also, RPerC increased the protein expression levels of eNOS, iNOS, $\mathrm{PKC} \varepsilon$, and $\mathrm{PKC} \delta$ in myocardium compared with control rats. These effects were blocked by naloxone, an opioid receptor antagonist, and glibenclamide, an ATP-sensitive $\mathrm{K}^{+}$channel blocker $\left(\mathrm{K}_{\mathrm{ATP}}\right)$. In summary, this study suggests that RPerC protects heart against $\mathrm{I} / \mathrm{R}$ injury through activation of opioid receptors and NO-PKC-K $\mathrm{K}_{\mathrm{ATP}}$ channel signaling pathways.

Key words: Remote ischemic per-conditioning, cardiac protection, ischemia/ reperfusion, opioid receptors, ATP-sensitive $\mathrm{K}^{+}$channels. 


\section{Introduction}

Coronary heart disease (CHD) is the leading cause of death all over the world. The major consequence of $\mathrm{CHD}$ is acute ischemia/reperfusion $(\mathrm{I} / \mathrm{R})$ injury in cardiac myocardium. Thus, development of innovative strategies to protect myocardium against I/R injury is necessary for patients with CHD.

Ischemic pre-conditioning (IPC) protects heart from cardiac tissue injury during prolonged ischemia through applying a short period of sub-lethal ischemia conducted in the heart (Murry et al. 1986). Ischemic post-conditioning (PostC), a modified schedule of reperfusion, also protects the heart from intermittent restoration of blood flow after a prolonged episode of ischemia (Zhao et al. 2003). Myocardial protection against $\mathrm{I} / \mathrm{R}$ can also be induced by ischemia in organs and tissues which are distant from the heart, a process called remote ischemic pre-conditioning (RIPC) (Aimo et al. 2015). RIPC is induced by a brief period of ischemia in distant organs such as limb (Birnbaum et al. 1997), kidney (Pell et al. 1998), or mesentery (Gho et al. 1996) to reduce myocardial infarction and protect against $\mathrm{I} / \mathrm{R}$ injury in porcine, rabbit, rat, as well as human (Kharbanda et al. 2002). RIPC is more attractive than traditional cardiac pre-conditioning or post-conditioning for its simple application in clinical practice and being the same efficiency as IPC or PostC. Like traditional cardiac conditioning, RIPC can be used before I/R. Also, it has been shown that several short periods of limb ischemia administered at the time of myocardial ischemia, termed as remote ischemia per-conditioning (RPerC), produce significant cardiac protection in pigs (Schmidt et al. 2007). Recently, Zhu et al compared three remote conditioning strategies on the cardiac protection in rats and found that RPerC produced more prevention of arrhythmia during I/R than that produced by RIPC or RIPostC (Zhu et al. 2013). The mechanism, however, for this phenomenon was not fully elucidated. 
The opioid receptors including $\mu, \delta$, and $\kappa$ subtypes and their endogenous ligands play important role in cardiac protection (Headrick et al. 2015). Many studies demonstrated that opioid receptors play important roles in the cardiac protection during IPC and PostC (Fraessdorf et al. 2015; Liu 2007; Lu et al. 2011). Also, opioid receptors are involved in the cardiac protection of RIPC (Rentoukas et al. 2010; Surendra et al. 2013; Wever et al. 2013; Wong et al. 2012). In this study, we determine whether opioid receptors are involved in the cardiac protection of RPerC.

The ATP-sensitive $\mathrm{K}^{+}$channels $\left(\mathrm{K}_{\mathrm{ATP}}\right)$ are activated by low concentration of intracellular ATP and are important $\mathrm{K}^{+}$channels that regulate myocardial function. $\mathrm{K}_{\mathrm{ATP}}$ channels are categorized into membrane $\mathrm{K}_{\mathrm{ATP}}$ channels and mitochondrion $\mathrm{K}_{\mathrm{ATP}}$ channels (mitoK $\left.\mathrm{K}_{\mathrm{ATP}}\right) . \mathrm{K}_{\mathrm{ATP}}$, especially mitoK $\mathrm{K}_{\mathrm{ATP}}$ channels, have been demonstrated to be involved in short-term cardiac protection such as ischemia pre-conditioning. In this regard, opening of the mitoK $_{\mathrm{ATP}}$ channels by diazoxide induces cardiac protection in nitric oxide (NO)-dependent manner (Wang et al. 2001b). Furthermore, Rakhit et al reported that the exogenous NO donor induces an early IPC through a cGMP-dependent mechanism and a delayed IPC by a cGMP-independent mechanism,

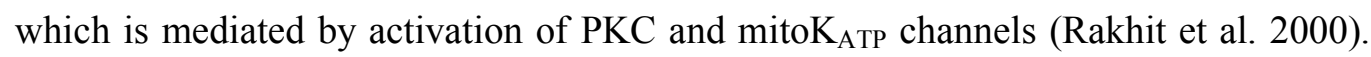
Thus, the cardiac protection of ischemia pre-conditioning is related with $\mathrm{K}_{\mathrm{ATP}}$, NO, and PKC. The aim of present study is to determine if RPerC protects heart against I/R injury through activation of opioid receptors via NO-PKC-mitoK $\mathrm{ATP}_{\mathrm{P}}$ signaling pathway.

\section{Materials and methods}

\section{Animals}

All animal experiments were conducted in compliance with the 1996 Guide for the 
Care and Use of Laboratory Animals (National Research Council, Bethesda, MD) and all procedures were reviewed and approved by the Ethics committee forthe Use of Experiment Animals at Hebei Medical University.

Adult male Sprague-Dawley rats (250-280g in body weight) were purchased from the experimental animal center of Hebei Medical University. These rats were divided randomly into five groups: control group, pre-conditioning group, per-conditioning group, naloxone group, and glibenclamide group. All rats were housed at room temperature with a natural light-dark cycle and had free access to standard rodent diet and tap water.

\section{Hemodynamics and left ventricular function measurement}

The rats were anesthetized with urethane (1g. $\mathrm{kg}^{-1}$, i.p.). After the trachea being cannulated, the rats were ventilated mechanically through rodent respirator $(1.0 \sim 1.5$ ml/100 g, 60 120 strokes/min, model 680A, Harvard Apparatus, Holliston, MA). The blood pressure (BP) was monitored through a pressure transducer with a tubing cannulated into the left femoral artery. The BP signals were fed into a PowerLab data acquisition system (AD Instrument, Australia). The right femoral artery was isolated for I/R conditioning and right femoral vein was cannulated for administration of drugs. A cannula was placed into the left cardiacventricle through the right carotid artery to record left ventricular systolic pressure (LVSP). The maximal rate of rise of left ventricular systolic pressure $\left(\mathrm{dp} / \mathrm{dt}_{\max }\right)$ and the maximal rate of decline of left ventricular systolic pressure $\left(\mathrm{dp} / \mathrm{dt}_{\mathrm{min}}\right)$ were recorded by differentiating LVSP. LVSP, $\mathrm{dp} / \mathrm{dt}_{\max }$ and $\mathrm{dp} / \mathrm{dt}_{\min }$ were used to assess the function of left ventricle. Rectal temperature was measured and kept at $37^{\circ} \mathrm{C}$ by using a servo-controlled heat blanket. Needle electrode was inserted subcutaneously in the legs and the standard lead II electrocardiography (ECG) was recorded throughout the experiment. The heart rate 
(HR) was calculated from ECG signal.

\section{Induction of myocardial I/R}

Myocardial ischemia was induced as described previously (Zhou et al. 2015). In brief, after thoracotomy and pericardiotomy, a thin silk suture (6.0 metric) was placed superficially into the myocardium around the left anterior descending (LAD) coronary artery $2 \mathrm{~mm}$ proximal from its origin in the atrio-ventricular sulcus. The suture was passed through small diameter tubing to forma snare, which was used to occlude the LAD coronary artery when it was tightened. The presence of myocardial ischemia was confirmed by elevation of the S-T segment in ECG and an immediate fall in blood pressure of $15-30 \mathrm{mmHg}$. For reperfusion, the snare was released, and reperfusion was visually confirmed by epicardial blushing, restoration of S-T segment in ECG gradually returned to the baseline level. A total of 30-min ischemia and 3-h reperfusion was performed. The changes of hemodynamics, left ventricular function and cardiac arrhythmia were recorded during I/R. The arrhythmia score was used to evaluate the degree of arrhythmia (Johnston et al. 1983; Walker et al. 1988).

\section{Assessment of myocardialinfarction}

At the end of reperfusion, the heart was removed quickly and frozen at $-20^{\circ} \mathrm{C}$. The frozen heart was cut into $1.5-\mathrm{mm}$ thin slices, which were perpendicular to the septum from the apex to the base. The myocardial slices were stained as described previously (Vivaldi et al. 1985). In brief, after 3-h reperfusion, 500 IU heparin was administered through the jugular vein to remove any clot in the heart. The LAD coronary artery was re-occluded, and $2 \mathrm{ml}$ of 1.5\% Evans Blue (Sigma, USA) was injected through the jugular vein to distinguish the non-ischemic area from area at risk (AAR). The heart was then excised, and the left ventricle was cut transversely into slices at $1-\mathrm{mm}$ thickness and incubated at $37^{\circ} \mathrm{C}$ with TTC (triphenyltetrazolium 
chloride; SD Fine Chemicals, Mumbai, India) solution (1\%inphosphatebuffer, $\mathrm{pH} 7.4$ ) for 30min. In the presence of dehydrogenase enzymes in the viable myocytes, NADH reacts with TTC to stain deep red while infarcted myocardium remains pale owing to the absence of dehydrogenases. The slices were fixed in $10 \%$ formalin for $6 \mathrm{~h}$ to obtain 3 colors: blue (non-ischemic area), deep and pale red (AAR as a whole), and unstained pale, representing the infarcted area (IA). The slices were sandwiched between 2 transparent slides and captured by using a scanner. The images were saved and analyzed in a blinded manner by computerized planimetry. Infarct size was expressed as the percentage of IA relative to AAR.

\section{Performance of pre-conditioning and per-conditioning}

RIPC was achieved by 5-min occlusion of right femoral artery followed by 5-min reperfusion and the occlusion-reperfusion was repeated for three times before LAD coronary artery occlusion. RPerC was performed during myocardial ischemia and before reperfusion. The femoral artery was exposed in the hind limb muscle where it joins the abdomen and isolated from the nerve and tissue in the pelvic region distal to any branch and occluded with a clip. During the ischemic period, the skin of the leg became purple and the temperature decreased. Reperfusionwas performed by removing the clip and was confirmed when the skin color returned to rose color and temperature increased to the level before ischemia.

\section{Western blot assay}

Western blotting was performed as described previously (Xi et al. 2002). Tissue samples from the myocardium were homogenized in ice-cold lysis buffer. The total proteins were extracted. After protein quantification, $50 \mu \mathrm{g}$ of total protein extracts were electrophoresed through polyacrylamide SDS gels and transferred by electroblotting onto PVDF membranes. Proteins were blocked with nonfat milk and 
incubated with primary rabbit polyclonal antibody against iNOS (dilution 1:500; Abcam), eNOS (dilution 1:2000; Abcam), PKC $\varepsilon$ (dilution 1:500; Abcam), and PKC $\delta$

(dilution 1:250; Abcam) at $4{ }^{\circ} \mathrm{C}$ overnight. The membranes were then incubated with a secondary horseradish peroxidase-conjugated anti-rabbit or anti-goat IgG (1:1000 or 2000 dilution; Amersham) for $1 \mathrm{~h}$ at room temperature. The blots were developed using the ECL system (immobilion TM Western, Millipore) and were analyzed by Quantity One Software (Bio-Rad, USA). Protein contents were normalized by glyceraldehyde-3-phosphate dehydrogenase (GAPDH, Proteintech) level. All experiments were repeated three times.

\section{Research protocols}

I. Rats in control group were subjected to 30-min ischemia (occlusion of LAD coronary artery) followed by 3-h reperfusion (re-opening the LAD coronary artery).

II. Rats in pre-conditioning group were subjected to remote pre-conditioning procedure: 5-min femoral artery occlusion followed by 1-min re-opening of the artery, which was repeated for three times before LAD coronary artery occlusion.

III. Rats in per-conditioninggroup were subjected to remote per-conditioning procedure: 5-min femoral artery occlusion followed by 1-min re-opening of the artery, which was repeated for three times during LAD coronary artery occlusion.

IV. Rats in naloxone group were received the same treatment as per-conditioning procedure and an injection of naloxone $\left(1.0 \mathrm{mg} . \mathrm{kg}^{-1}\right.$, Sigma), an antagonist of opioid receptors, 5 min before per-conditioning procedure.

$\mathrm{V}$. Rats in glibenclamide group were received the same treatment as per-conditioning procedure and an injection of glibenclamide (1.0 mg. $\mathrm{kg}^{-1}$, Sigma), an antagonist of $\mathrm{K}_{\mathrm{ATP}}$ channels, 5 min before per-conditioning procedure.

\section{Statistics analysis}


All data were expressed as mean \pm SEM. Differences in infarct sizes, biochemical values, and hemodynamics were compared by one-way ANOVA followed by Tukey-Krame post hoc test for multiple comparisons. The comparison between same groups was made by student $t$ test. Differences were considered significant at $P$ values less than 0.05 .

\section{Results}

\section{Effect of RPerC on hemodynamics}

The baseline systolic artery pressure (SAP), diastolic artery pressure (DAP) and heart rate (HR) showed no differences among groups. Cardiac ischemia decreased SAP significantly $(P<0.05-0.01$, Table1). However, pre-conditioning procedure attenuated the depressor effect induced by ischemia. In the following reperfusion phase, SAP still remained at lower level. Pre-conditioning procedure and per-conditioning procedure restored the SAP to baseline level from I/R-induced depressor response $(P<0.05-0.01)$. After 3 -h reperfusion, SAP was significantly higher in pre-conditioning rats than in control rats $(P<0.05$, Table 1$)$. DAP was not changed during whole process of $\mathrm{I} / \mathrm{R}$ in all groups $(P>0.05$, Table 2$)$.

There was no significant difference of HR during ischemia among all groups $(P>0.05)$. During reperfusion phase, HR was significantly decreased in control, naloxone, and glibenclamide rats compared with pre-conditioning and per-conditioning rats, respectively $(P<0.05$, Table 3$)$.

\section{Effect of RPerC on left ventricular function}

The parameters of left ventricular function were similar at basal condition among groups $(P>0.05)$. Ischemia procedure caused significant decreases in LVSP, $\mathrm{dp} / \mathrm{dt}_{\max }$ 
and $\mathrm{dp} / \mathrm{dt}_{\min }$. Per-conditioning or pre-conditioning treatment significantly attenuated ischemia-induced reduction of LVSP, $\mathrm{dp} / \mathrm{dt}_{\max }$ and $\mathrm{dp} / \mathrm{dt}_{\min }$. However, naloxone or glibenclamide injection eliminated the effects of per-conditioning and preconditioning treatment on these parameters. During reperfusion phase, LVSP, $\mathrm{dp} / \mathrm{dt}_{\max }$ and $\mathrm{dp} / \mathrm{dt}_{\min }$ were partly recovered gradually. The degree of recovery for LVSP, $d p / d_{\max }$ and $\mathrm{dp} / \mathrm{dt}_{\min }$ was higher in per-conditioning and pre-conditioning groups than those in control, naloxone, or glibenclamide groups $(P<0.05-0.01)$. In addition, the left ventricular function during $\mathrm{I} / \mathrm{R}$ did not differ between control, naloxone or glibenclamide-treated rats $(P>0.05$, Figure 1).

\section{Effect of RPerC on cardiac infarct size}

After cardiacI/R, the ratios of infarct area to risk area were $42.0,12.2,11.5,31.4$ and $45.6 \%$ in control, pre-conditioning, per-conditioning, naloxone and glibenclamide rats, respectively. The ratio in per-conditioning rats was significantly smaller than in control, naloxone, or glibenclamide rats $(P<0.05-0.01)$, but did not differ compared with pre-conditioning rats $(P>0.05$, Figure 2$)$.

\section{Effect of RPerC on ventricular arrhythmia}

Arrhythmia including ventricular premature heartbeat, tachycardia, and fibrillation was induced by I/R procedure. The arrhythmia scores were $2.9,1.5,1.4$, 2.1, and 3.0 in control, pre-conditioning, per-conditioning, naloxone and glibenclamide rats during ischemia and 4.1, 1.6, 2.0, 2.8, and 3.4 during reperfusion. The scores in pre-conditioningrats were significantly smaller than those in control, naloxone, and glibenclamide rats $(P<0.05$, Figure 3$)$, but did not differ compared with per-conditioning rats during $\mathrm{I} / \mathrm{R}(P>0.05$, Figure 3$)$. These results suggest that per-conditioning and pre-conditioning protected heart against I/R injury, and the protective effect was eliminated by pretreatment with naloxone or glibenclamide. 


\section{Effect of RPerC on expression of NOS in myocardium}

Compared with controlrats, the expression of iNOS and eNOS in per-conditioning rats was increased significantly $(P<0.01)$. The expression of iNOS was higher in naloxone or glibenclamide rats than in control rats, but was lower than in per-conditioning rats $(P<0.05)$. The expression levels of eNOS did not differ among control, naloxone, or glibenclamide rats $(P>0.05$, Figure4).

\section{Effect of RPerCon expression of PKC in myocardium}

Compared with control rat, $\mathrm{PKC} \varepsilon$ and $\mathrm{PKC} \delta$ expression levels in per-conditioning rats were increased significantly $(P<0.01)$. The expression of $\mathrm{PKC} \varepsilon$ was similar among control, naloxone, or glibenclamide rats $(P>0.05)$. The expression of $\mathrm{PKC} \delta$ in naloxone rats was increased compared with control rats, but decreased compared with per-conditioning rats $(P<0.05$, Figure 5).

\section{Discussion}

In the present study, we examined the cardiac protective effect of RPerC during I/R. We also compared the protective effect of RPerC with that generated by RIPC. We found that RPerC, like RIPC, induced cardiac protection against I/R injury effectively. RPerC or RIPC procedure significantly improved the recovery of left ventricular function and reduced I/R-induced myocardial infarction. Our findings demonstrated that RPerC has significant cardiac protection against I/R injury. Unlike Zhu's report that RPerC induces much better cardiac protection than RIPC or RIPostC does (Zhu et al. 2013), our study demonstrated that RPerC produced the same level of protective effects against cardiac I/R injury as RIPC does. Furthermore, we found that the cardiac protective effect of RPerC was eliminated by naloxone, a blocker of opioid receptors, or glibenclamide, a blocker of $\mathrm{K}_{\text {ATP }}$ channels. These data suggest that opioid receptors and $\mathrm{K}_{\mathrm{ATP}}$ channels are involved in the cardiac protection produced by RPerC 
treatment.

Naloxone, a specific antagonist for opioid receptors, antagonizes the effect generated by both exogenous and endogenous opioids. Opioid receptors are divided into four sub-types: $\mu, \delta, \kappa$, and $\sigma$ subtypes. The $\mu, \delta$, and $\kappa$ opioid subunits are distributed in human and rodent heart tissues. Only $\delta$ and $\kappa$ subunits are found in adult rat heart. It has been shown that cardiac protection of RIPC isrelated to opioid signaling pathways (Patel et al. 2002). Furthermore, cardiac protection of RIPC is closely related to $\delta$ opioid receptor, but not $\mu$ - or $\kappa$-opioid receptors (Schultz et al. 1998). Previous study has shown that $\kappa$-opioid receptors mediate the improvement of ischemia-induced infarction and arrhythmia through PKC and $\mathrm{K}_{\mathrm{ATP}}$ pathways, while $\delta$-opioid receptors are involved in improvement of ischemia-induced infarction (Wang et al. 2001a). It has been shown that $\mathrm{K}_{\mathrm{ATP}}$ channels mediate the cardiac protection of $\delta$-receptor. Activation of $\mathrm{K}_{\text {ATP }}$ channels inhibits $\mathrm{Ca}^{2+}$ influx to reduce $\mathrm{Ca}^{2+}$ overload in myocardium and leads to an alleviation of myocardium injury (Schultz et al. 1997). However, whether opioid receptor system is involved in the cardiac protection of RPerC is not previously known. In this study, we found that the cardiac protection of RPerC was abolished by naloxone, suggesting that the opioid receptorsare involved in the cardiac protection of RPerC. However, we did not identify the receptor subtypes, which are involved in RIPC or RPerC. Thus, further studies are needed to identify the subtype of opioid receptors involved in cardiac protection by RIPC or RPerC (Maslov et al. 2016).

$\mathrm{K}_{\mathrm{ATP}}$ channels play an important role in the cardiac protection under pathological situations. It has been reported that $\mathrm{K}_{\text {ATP }}$ channel openers suppress cardiac arrhythmia after ischemia reperfusion (Watanabe et al. 2011). Also, overexpression of $\mathrm{K}_{\mathrm{ATP}}$ subunit increases resistance against cardiac ischemia (Du et al. 2006). Previous 
studies focusing on mechanisms of remote pre- or post-conditioning have revealed that $\mathrm{K}_{\text {ATP }}$ channels also contribute to cardiac protection in remote ischemia conditioning (Loukogeorgakis et al. 2007). In addition, mitoK $_{\text {ATP }}$ channels play roles in cardiac protection of pre-conditioning and post-conditioning (Das and Sarkar 2005; Penna et al. 2006). Thus, it is likely that opening of $\mathrm{K}_{\mathrm{ATP}}$ channels, especially mitoK $\mathrm{K}_{\mathrm{ATP}}$ channels, serve as a common mechanism to prevent myocardium from I/R injury in variety of conditionings. Our study found that glibenclamide completely abolished the protective effect of RPerC in rats, suggesting that $\mathrm{K}_{\mathrm{ATP}}$ channels in sarcolemma or mitochondria membrane participate in cardiac protection of RPerC against I/R injury.

It is well known that enhanced bioavailability of endogenous nitric oxide (NO) produced by the NO-synthases (NOS) mediates cardiac protection against I/R-induced injury. Both in vivo and in vitro studies have demonstrated that pharmacological inhibition of NO synthesis or genetic ablation of constitutive endothelial NOS (eNOS) exerts detrimental effects during myocardial infarction. On the other hand, NO donors or overexpression of eNOS or iNOS reduce myocardial injury (Jones et al. 2004; Jugdutt 2002; Kanno et al. 2000; Xuan et al. 2007). In this study, we found that the expression levels of eNOS and iNOS were increased in RPerC rats compared with control rats, suggesting that NO is involved in the cardiac protection produced by RPerC.

Previous studies have shown that opioid, $\mathrm{PKC}$, mitoK $_{\mathrm{ATP}}$ channels and $\mathrm{NO}$ in IPC or RIPC interact (Cohen and Downey 2015; Maslov et al. 2016). Liu et al. reported that IPC is triggered by receptor occupancy (Liu et al. 1991). Schultz et al. reported that opioid, an endogenously released substances during $\mathrm{I} / \mathrm{R}$, binds to its receptors (Gprotein-coupled receptor) and the second messenger $\mathrm{G}_{\mathrm{i}}$ protein is cleaved into active $\alpha$ and $\beta \gamma$ subunits which then results in activation of PKC (Schultz et al. 
1995). After being activated and translocated to the cell membrane, PKC phosphorylates multiple intracellular substrates and leads to the opening of the mitoK $_{\text {ATP }}$ channels (Downey et al. 2007; Schulz et al. 2001). The opening of mitoK ${ }_{\text {ATP }}$ channel is a critical step in cardiac protection signaling cascade. Opening of mitoK $\mathrm{K}_{\mathrm{ATP}}$ blocks the mitochondrial permeability transition pore (MPTP) and inhibits apoptosis (Costa and Garlid 2008). IPC's end-effector appears to be the MPTP and the inhibition of MPTP is considered to be the final step in the protective signal transduction pathway of pre-conditioning (Di Lisa et al. 2001; Hausenloy et al. 2002). Recent studies have shown that PKCe translocation may be a downstream event of mitoK $_{\text {ATP }}$ channels (Wang et al. 2015). It is possible that there is feedback regulation between PKC and mitoK $\mathrm{ATP}_{\mathrm{A}}$ channels. Studies on cardiac protection induced by IPC showed that activation of PKC leads to the opening of the mitoK $\mathrm{ATP}_{\mathrm{P}}$ channels which triggers the modest ROS formation and then activates PKC (Downey et al. 2007). NO is an important biological regulator and cellular signaling molecule. The eNOS has been implicated in the preconditioning pathways between $\mathrm{Gi} / \mathrm{o}$-coupled receptors and PKC (Maslov et al. 2016). Thus, we propose that opioid produced during ischemia in RPerC may activate NOS, PKC, and mitoK $_{\mathrm{ATP}}$ channels and inhibit MPTP to produce cardiac protection. The blockade of any elementsin this signaling pathway may result in a loss of cardiac protection.

In summary, this study demonstrated for the first time that RPerC protects heart against I/R injury through activation opioid receptor system and NO-PKC-mitoK $\mathrm{ATP}_{\mathrm{P}}$ channel signaling pathways.

\section{Acknowledgements}

This study was supported by the National Basic Research Development Program of 
China (No. 2012CB518200). 


\section{References}

Aimo, A., Borrelli, C., Giannoni, A., Pastormerlo, L.E., Barison, A., Mirizzi, G., et al. 2015. Cardioprotection by remote ischemic conditioning: Mechanisms and clinical evidences. World J. Cardiol. 7(10): 621-632. doi: 10.4330/wjc.v7.i10.621.

Birnbaum, Y., Hale, S.L., and Kloner, R.A. 1997. Ischemic preconditioning at a distance: reduction of myocardial infarct size by partial reduction of blood supply combined with rapid stimulation of the gastrocnemius muscle in the rabbit. Circulation 96(5): 1641-1646.

Cohen, M.V., and Downey, J.M. 2015. Signalling pathways and mechanisms of protection in pre- and postconditioning: historical perspective and lessons for the future. Br.J. pharmacol.172(8): 1913-1932. doi: 10.1111/bph.12903.

Costa, A.D., and Garlid, K.D. 2008. Intramitochondrial signaling: interactions among mitoKATP, PKC epsilon, ROS, and MPT. Am. J. physiol.: Heart Circ. Physiol. 295(2): H874-882. doi: 10.1152/ajpheart.01189.2007.

Das, B., and Sarkar, C. 2005. Is the sarcolemmal or mitochondrial K(ATP) channel activation important in the antiarrhythmic and cardioprotective effects during acute ischemia/reperfusion in the intact anesthetized rabbit model? Life Sci.77(11): 1226-1248. doi: 10.1016/j.1fs.2004.12.042.

Di Lisa, F., Menabo, R., Canton, M., Barile, M., and Bernardi, P. 2001. Opening of the mitochondrial permeability transition pore causes depletion of mitochondrial and cytosolic $\mathrm{NAD}+$ and is a causative event in the death of myocytes in postischemic reperfusion of the heart. J.Biol.Chem. 276(4): 2571-2575. doi: 10.1074/jbc.M006825200.

Downey, J.M., Davis, A.M., and Cohen, M.V. 2007. Signaling pathways in ischemic preconditioning. Heart Failure Rev.12(3-4): 181-188. doi: 10.1007/s10741-007-9025-2.

Du, Q., Jovanovic, S., Clelland, A., Sukhodub, A., Budas, G., Phelan, K., et al. 2006. Overexpression of SUR2A generates a cardiac phenotype resistant to ischemia. FASEB J. 20(8): 1131-1141. doi: 10.1096/fj.05-5483com.

Fraessdorf, J., Hollmann, M.W., Hanschmann, I., Heinen, A., Weber, N.C., Preckel, B., et al. 2015. Role of Endogenous Opioid System in Ischemic-Induced Late Preconditioning. PLoS One 10(7): e0134283. doi: 10.1371/journal.pone.0134283.

Gho, B.C., Schoemaker, R.G., van den Doel, M.A., Duncker, D.J., and Verdouw, P.D. 1996. Myocardial protection by brief ischemia in noncardiac tissue. Circulation 94(9): 2193-2200.

Hausenloy, D.J., Maddock, H.L., Baxter, G.F., and Yellon, D.M. 2002. Inhibiting mitochondrial permeability transition pore opening: a new paradigm for myocardial preconditioning? Cardiovasc. Res. 55(3): 534-543.

Headrick, J.P., See Hoe, L.E., Du Toit, E.F., and Peart, J.N. 2015. Opioid receptors and cardioprotection - 'opioidergic conditioning' of the heart. Br. J. Pharmacol. 172(8): 2026-2050. doi: 10.1111/bph.13042.

Johnston, K.M., MacLeod, B.A., and Walker, M.J. 1983. Responses to ligation of a coronary artery in conscious rats and the actions of antiarrhythmics. Can. J. Physiol. Pharmacol. 61(11): 1340-1353.

Jones, S.P., Greer, J.J., Kakkar, A.K., Ware, P.D., Turnage, R.H., Hicks, M., et al. 2004. Endothelial nitric oxide synthase overexpression attenuates myocardial reperfusion injury. 
Am. J. physiol.: Heart Circ.Physiol. 286(1): H276-282. doi: 10.1152/ajpheart.00129.2003.

Jugdutt, B.I. 2002. Nitric oxide and cardioprotection during ischemia-reperfusion. Heart Failure Rev. 7(4): 391-405.

Kanno, S., Lee, P.C., Zhang, Y., Ho, C., Griffith, B.P., Shears, L.L., et al. 2000. Attenuation of myocardial ischemia/reperfusion injury by superinduction of inducible nitric oxide synthase. Circulation 101(23): 2742-2748.

Kharbanda, R.K., Mortensen, U.M., White, P.A., Kristiansen, S.B., Schmidt, M.R., Hoschtitzky, J.A., et al. 2002. Transient limb ischemia induces remote ischemic preconditioning in vivo. Circulation 106(23): 2881-2883.

Liu, G.S., Thornton, J., Van Winkle, D.M., Stanley, A.W., Olsson, R.A., and Downey, J.M. 1991. Protection against infarction afforded by preconditioning is mediated by A1 adenosine receptors in rabbit heart. Circulation 84(1): 350-356.

Liu, X.H. 2007. [Progress in endogenous cardioprotection induced by ischemic postconditioning]. Sheng Li Xue Bao 59(5): 628-634.

Loukogeorgakis, S.P., Williams, R., Panagiotidou, A.T., Kolvekar, S.K., Donald, A., Cole, T.J., et al. 2007. Transient limb ischemia induces remote preconditioning and remote postconditioning in humans by a K(ATP)-channel dependent mechanism. Circulation 116(12): 1386-1395. doi: 10.1161/CIRCULATIONAHA.106.653782.

Lu, Y., Dong, C., Yu, J., and Li, L. 2011. Role of central and peripheral opioid receptors in the cardioprotection of intravenous morphine preconditioning. Ir. J. Med. Sci. 180(4): 881-885. doi: 10.1007/s11845-011-0734-0.

Maslov, L.N., Khaliulin, I., Oeltgen, P.R., Naryzhnaya, N.V., Pei, J.M., Brown, S.A., et al. 2016. Prospects for Creation of Cardioprotective and Antiarrhythmic Drugs Based on Opioid Receptor Agonists. Med. Res. Rev. 36(5): 871-923. doi: 10.1002/med.21395.

Murry, C.E., Jennings, R.B., and Reimer, K.A. 1986. Preconditioning with ischemia: a delay of lethal cell injury in ischemic myocardium. Circulation 74(5): 1124-1136.

Patel, H.H., Moore, J., Hsu, A.K., and Gross, G.J. 2002. Cardioprotection at a distance: mesenteric artery occlusion protects the myocardium via an opioid sensitive mechanism. J. Mol. Cell. Cardiol. 34(10): 1317-1323.

Pell, T.J., Baxter, G.F., Yellon, D.M., and Drew, G.M. 1998. Renal ischemia preconditions myocardium: role of adenosine receptors and ATP-sensitive potassium channels. Am. J. Physiol 275(5 Pt 2): H1542-1547.

Penna, C., Rastaldo, R., Mancardi, D., Raimondo, S., Cappello, S., Gattullo, D., et al. 2006. Post-conditioning induced cardioprotection requires signaling through a redox-sensitive mechanism, mitochondrial ATP-sensitive $\mathrm{K}+$ channel and protein kinase $\mathrm{C}$ activation. Basic Res. Cardiol. 101(2): 180-189. doi: 10.1007/s00395-006-0584-5.

Rakhit, R.D., Edwards, R.J., Mockridge, J.W., Baydoun, A.R., Wyatt, A.W., Mann, G.E.et al. 2000. Nitric oxide-induced cardioprotection in cultured rat ventricular myocytes. Am. J. physiol.: Heart Circ.Physiol. 278(4): H1211-1217.

Rentoukas, I., Giannopoulos, G., Kaoukis, A., Kossyvakis, C., Raisakis, K., Driva, M. et al. 2010. Cardioprotective role of remote ischemic periconditioning in primary percutaneous coronary intervention: enhancement by opioid action. JACC Cardiovasc. Interv. 3(1): 49-55. doi: 10.1016/j.jcin.2009.10.015.

Schmidt, M.R., Smerup, M., Konstantinov, I.E., Shimizu, M., Li, J., Cheung, M. et al. 2007. 
Intermittent peripheral tissue ischemia during coronary ischemia reduces myocardial infarction through a KATP-dependent mechanism: first demonstration of remote ischemic perconditioning. Am. J. physiol.: Heart Circ.Physiol. 292(4): H1883-1890. doi: 10.1152/ajpheart.00617.2006.

Schultz, J.E., Hsu, A.K., and Gross, G.J. 1998. Ischemic preconditioning in the intact rat heart is mediated by delta1- but not mu- or kappa-opioid receptors. Circulation 97(13): 1282-1289.

Schultz, J.E., Rose, E., Yao, Z., and Gross, G.J. 1995. Evidence for involvement of opioid receptors in ischemic preconditioning in rat hearts. Am. J. physiol. 268(5 Pt 2): H2157-2161.

Schultz, J.J., Hsu, A.K., and Gross, G.J. 1997. Ischemic preconditioning and morphine-induced cardioprotection involve the delta (delta)-opioid receptor in the intact rat heart. J. Mol. Cell. Cardiol. 29(8): 2187-2195. doi: 10.1006/jmcc.1997.0454.

Schulz, R., Cohen, M.V., Behrends, M., Downey, J.M., and Heusch, G. 2001. Signal transduction of ischemic preconditioning. Cardiovasc. Res. 52(2): 181-198.

Surendra, H., Diaz, R.J., Harvey, K., Tropak, M., Callahan, J., Hinek, A., et al. 2013. Interaction of delta and kappa opioid receptors with adenosine A1 receptors mediates cardioprotection by remote ischemic preconditioning. J. Mol. Cell. Cardiol. 60: 142-150. doi: 10.1016/j.yjmcc.2013.04.010.

Vivaldi, M.T., Kloner, R.A., and Schoen, F.J. 1985. Triphenyltetrazolium staining of irreversible ischemic injury following coronary artery occlusion in rats. Am. J.Pathol. 121(3): 522-530.

Walker, M.J., Curtis, M.J., Hearse, D.J., Campbell, R.W., Janse, M.J., Yellon, D.M., et al. 1988. The Lambeth Conventions: guidelines for the study of arrhythmias in ischaemia infarction, and reperfusion. Cardiovasc. Res. 22(7): 447-455.

Wang, C., Hu, S.M., Xie, H., Qiao, S.G., Liu, H., and Liu, C.F. 2015. Role of mitochondrial ATP-sensitive potassium channel-mediated PKC-epsilon in delayed protection against myocardial ischemia/reperfusion injury in isolated hearts of sevoflurane-preconditioned rats. Braz. J. Med. Bio. Res. 48(6): 528-536. doi: 10.1590/1414-431X20143876.

Wang, G.Y., Wu, S., Pei, J.M., Yu, X.C., and Wong, T.M. 2001a. Kappa- but not delta-opioid receptors mediate effects of ischemic preconditioning on both infarct and arrhythmia in rats. Am. J. physiol.: Heart Circ.Physiol. 280(1): H384-391.

Wang, Y., Kudo, M., Xu, M., Ayub, A., and Ashraf, M. 2001b. Mitochondrial K(ATP) channel as an end effector of cardioprotection during late preconditioning: triggering role of nitric oxide. J. Mol. Cell. Cardiol. 33(11): 2037-2046. doi: 10.1006/jmcc.2001.1468.

Watanabe, I., Okumura, Y., Ohkubo, K., Nagashima, K., Mano, H., Sonoda, K., et al. 2011. Effect of the ATP-sensitive $\mathrm{K}(+)$ channel opener nicorandil in a canine model of proarrhythmia. Int. Heart J. 52(5): 318-322.

Wever, K.E., Masereeuw, R., Wagener, F.A., Verweij, V.G., Peters, J.G., Pertijs, J.C., et al. 2013. Humoral signalling compounds in remote ischaemic preconditioning of the kidney, a role for the opioid receptor. Nephrol. Dial. Transplant. 28(7): 1721-1732. doi: 10.1093/ndt/gfs601.

Wong, G.T., Lu, Y., Mei, B., Xia, Z., and Irwin, M.G. 2012. Cardioprotection from remote preconditioning involves spinal opioid receptor activation. Life Sci. 91(17-18): 860-865. 
doi: 10.1016/j.1fs.2012.08.037.

Xi, L., Tekin, D., Gursoy, E., Salloum, F., Levasseur, J.E., and Kukreja, R.C. 2002. Evidence that NOS2 acts as a trigger and mediator of late preconditioning induced by acute systemic hypoxia. Am. J. physiol.: Heart Circ.Physiol. 283(1): H5-12. doi: 10.1152/ajpheart. 00920.2001.

Xuan, Y.T., Guo, Y., Zhu, Y., Wang, O.L., Rokosh, G., and Bolli, R. 2007. Endothelial nitric oxide synthase plays an obligatory role in the late phase of ischemic preconditioning by activating the protein kinase $\mathrm{C}$ epsilon $\mathrm{p} 44 / 42$ mitogen-activated protein kinase $\mathrm{pSer}$-signal transducers and activators of transcription $1 / 3$ pathway. Circulation 116(5): 535-544. doi: 10.1161/CIRCULATIONAHA.107.689471.

Zhao, Z.Q., Corvera, J.S., Halkos, M.E., Kerendi, F., Wang, N.P., Guyton, R.A., et al. 2003. Inhibition of myocardial injury by ischemic postconditioning during reperfusion: comparison with ischemic preconditioning. Am. J. physiol.: Heart Circ.Physiol. 285(2): H579-588. doi: 10.1152/ajpheart.01064.2002.

Zhou, J.J., Ma, H.J., Liu, Y., Guan, Y., Maslov, L.N., Li, D.P., et al. 2015. The anti-arrhythmic effect of chronic intermittent hypobaric hypoxia in rats with metabolic syndrome induced with fructose. Can. J. Physiol. Pharmacol. 93(4): 227-232. doi: 10.1139/cjpp-2014-0343.

Zhu, S.B., Liu, Y., Zhu, Y., Yin, G.L., Wang, R.P., Zhang, Y., et al. 2013. Remote preconditioning, perconditioning, and postconditioning: a comparative study of their cardio-protective properties in rat models. Clinics (Sao Paulo) 68(2): 263-268. 


\section{Tables}

Table 1. Effect of per-conditioning on systolic arterial pressure during $\mathrm{I} / \mathrm{R}$ in rats

\begin{tabular}{lcccccc}
\hline & Baseline & Ischemia & Reperf-15min & Reperf-1h & Reperf-2h & Reperf-3h \\
& $(\mathrm{mmHg})$ & $(\mathrm{mmHg})$ & $(\mathrm{mmHg})$ & $(\mathrm{mmHg})$ & $(\mathrm{mmHg})$ & $(\mathrm{mmHg})$ \\
\hline CON & $110.7 \pm 7.4$ & $90.6 \pm 5.7 \#$ & $92.4 \pm 5.2 \#$ & $91.6 \pm 7.1 \#$ & $88.8 \pm 7.4 \#$ & $81.3 \pm 8.6 \#$ \\
& & & & & \\
Pre-C & $114.6 \pm 9.4$ & $98.9 \pm 6.5$ & $101.3 \pm 7.9$ & $103.0 \pm 9.5$ & $95.9 \pm 10.1$ & $92.4 \pm 10.6$ \\
& & & & & $103.0 \pm 4.5$ & $105.8 \pm 4.0 *$ \\
Per-C & $109.6 \pm 2.9$ & $90.5 \pm 5.7 \#$ & $95.3 \pm 7.9$ & $97.6 \pm 6.0$ & & $101.2 \pm 6.9 \#$ \\
Nal & $117.6 \pm 3.0$ & $97.0 \pm 7.9 \#$ & $100.6 \pm 7.0 \#$ & $99.9 \pm 6.4 \#$ & $101.3 \pm 7.0 \#$ & \\
& & & & & & \\
Gli & $116.8 \pm 3.1$ & $90.4 \pm 7.2 \# \#$ & $93.3 \pm 2.9 \# \#$ & $100.2 \pm 2.5 \# \#$ & $100.8 \pm 4.4 \#$ & $100.9 \pm 5.3 \#$ \\
\hline
\end{tabular}

CON: control group; Pre-C:pre-conditioning group; Per-C: per-conditioning group; Nal: Naloxone group; Gli: glibenclamidegroup; Reperf-: reperfusion; All data were expressed as Mean \pm SEM, $n=8$ for each group, ${ }^{*} P<0.05$ vs $\mathrm{CON} \# P<0.05 \# \# P<0.01$ vs Baseline

Table 2. Effect of per-conditioning on diastolic artery pressure during I/R in rats

\begin{tabular}{lcccccc}
\hline & Baseline & Ischemia & Reperf-15min & Reperf-1h & Reperf-2h & Reperf-3h \\
& $(\mathrm{mmHg})$ & $(\mathrm{mmHg})$ & $(\mathrm{mmHg})$ & $(\mathrm{mmHg})$ & $(\mathrm{mmHg})$ & $(\mathrm{mmHg})$ \\
\hline CON & $52.6 \pm 3.8$ & $49.3 \pm 4.8$ & $45.9 \pm 3.9$ & $44.6 \pm 5.8$ & $49.8 \pm 6.1$ & $45.7 \pm 7.7$ \\
& & & & & \\
Pre-C & $61.4 \pm 5.3$ & $53.0 \pm 2.7$ & $52.5 \pm 4.3$ & $55.2 \pm 5.2$ & $52.1 \pm 5.5$ & $46.8 \pm 6.1$ \\
& & & & & \\
Per-C & $57.2 \pm 5.1$ & $56.8 \pm 4.5$ & $59.2 \pm 5.6$ & $58.2 \pm 4.0$ & $63.4 \pm 7.4$ & $60.4 \pm 5.8$ \\
Nal & $62.0 \pm 3.5$ & $56.3 \pm 4.7$ & $56.2 \pm 4.9$ & $58.1 \pm 3.7$ & $58.9 \pm 4.1$ & $59.8 \pm 4.2$ \\
& & & & & \\
Gli & $65.4 \pm 7.4$ & $59.3 \pm 7.9$ & $56.2 \pm 5.5$ & $60.1 \pm 4.9$ & $60.4 \pm 6.8$ & $60.2 \pm 7.4$ \\
\hline
\end{tabular}

CON: control group; Pre-C: preconditioning group; Per-C: per-conditioning group; Nal: Naloxone group; Gli: glibenclamide group; Reperf-: reperfusion; All data were expressed as Mean \pm SE, $n=8$ for each group 
Table 3. Effect of per-conditioning on heart rate during $\mathrm{I} / \mathrm{R}$ in rats

\begin{tabular}{lcccccc}
\hline & Baseline & Ischemia & Reperf-15min & Reperf-1h & Reperf-2h & Reperf-3h \\
& $(\mathrm{mmHg})$ & $(\mathrm{mmHg})$ & $(\mathrm{mmHg})$ & $(\mathrm{mmHg})$ & $(\mathrm{mmHg})$ & $(\mathrm{mmHg})$ \\
\hline CON & $374.7 \pm 18.0$ & $361.0 \pm 8.0$ & $321.5 \pm 29.6$ & $313.9 \pm 13.6 \#$ & $321.6 \pm 9.4 \#$ & $311.6 \pm 7.4 \# \#$ \\
& & & & & & \\
Pre-C & $384.2 \pm 20.8$ & $363.3 \pm 28.5$ & $351.5 \pm 24.8$ & $347.7 \pm 16.4$ & $337.4 \pm 11.3$ & $332.7 \pm 16.0$ \\
& & & & & & \\
Per-C & $377.5 \pm 13.6$ & $373.6 \pm 12.6$ & $362.4 \pm 11.9$ & $344.2 \pm 12.2$ & $337.4 \pm 11.8$ & $334.4 \pm 12.2$ \\
Nal & $388.6 \pm 8.7$ & $358.8 \pm 12.6$ & $315.8 \pm 11.7 \# \#$ & $334.3 \pm 10.8 \# \#$ & $325.2 \pm 8.1 \# \#$ & $326.4 \pm 10.0 \# \#$ \\
& & & & & & \\
Gli & $370.9 \pm 18.6$ & $325.3 \pm 15.7$ & $312.1 \pm 15.2 \#$ & $308.8 \pm 10.1 \#$ & $307.4 \pm 8.7 \# \#$ & $299.9 \pm 7.1 \# \#$ \\
\hline
\end{tabular}

CON: control group; Pre-C: Preconditioning group; Per-C: per-conditioning group; Nal: Naloxone group; Gli: glibenclamide group; Reperf-: reperfusion; All data were expressed as Mean \pm SEM, $n=8$ for each group, $\# P<0.05$ $\# \# P<0.01$ vs Baseline 


\section{Figurelegend}

Figure 1.Effect of RPerC on left ventricular function. Data were expressed as Mean \pm SEM, $n=8$ for each group $* P<0.05 * * P<0.01$ vsCON\#P<0.05 \#\# $P<0.01$ vs Pre-C $+P<0.05++P<0.01$ vs Per-C. LVSP: left ventricular systolic pressure; $\mathrm{dp} / \mathrm{dt}_{\max }$ : maximal rate of rise of left ventricular systolic pressure; $\mathrm{dp} / \mathrm{dt}_{\min }$ : maximal rate of decline of left ventricular systolic pressure; $\mathrm{CON}$ : control group; Pre-C: pre-conditioning group; Per-C: per-conditioning group; Nal: naloxone group; Gli: glibeclamide group.

Figure 2.Effect of RPerC on myocardial infarct size. A. The representative staining of myocardium in rats. B. Myocardial infarct sizein different groups. Blue stands for non-ischemic area; Deep and pale red represents area at risk; Unstained pale represents the infarcted area. Data were expressed as Mean \pm SEM, $n=4$ for each group * $P<0.05 * * P<0.01$ vs $\mathrm{CON} \# P<0.05 \# \# P<0.01$ vs Per-C.CON: control group; Pre-C: pre-conditioning group; Per-C: per-conditioning group; Nal: Naloxone group; Gli: glibeclamide group;

Figure 3.Effect of RPerC on arrhythmia induced by $\mathrm{I} / \mathrm{R}$ in rat. Data were expressed as Mean \pm SE, $n=8$ for each group $* P<0.05 * * P<0.01$ s CON, $\# P<0.05$ vs Per-C. AS: arrhythmia score; CON: control group; Pre-C: pre-conditioning group; Per-C: per-conditioning group; Nal: Naloxone group; Gli: glibeclamide group.

Figure 4.Effect of RPerC on expression of NOS in myocardium. Data were expressed as Mean \pm SEM, $n=4$ for each group. ${ }^{*} * P<0.01$ vs CON, \#\# $P<0.01$ vs Per-C.CON: control group; Pre-C: pre-conditioning group; Per-C: per-conditioning group; Nal: Naloxone group; Gli: glibeclamide group.

Figure 5.Effect of RPerC on expression of PKC in myocardium. Data were expressed as Mean \pm SEM, $\mathrm{n}=4$ for each group ** $\mathrm{P}<0.01$ vs $\mathrm{CON}$, \#\# $\mathrm{P}<0.01$ vs Per-C.CON: control group; Pre-C: pre-conditioning group; Per-C: per-conditioning group; Nal: Naloxone group; Gli: glibeclamide group. 

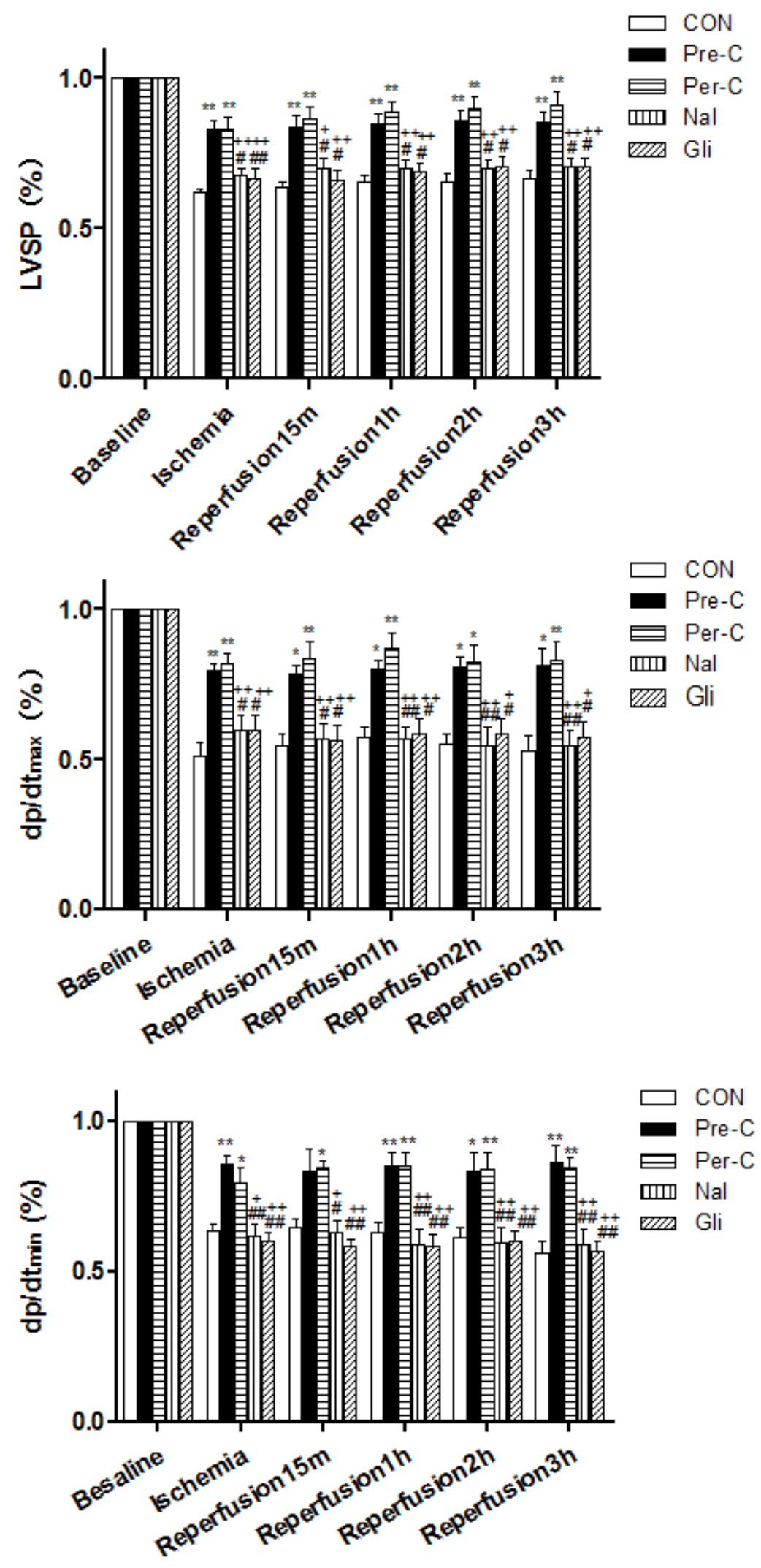

https://mc06.manuscriptcentral.com/cjpp-pubs 
A

CON/R
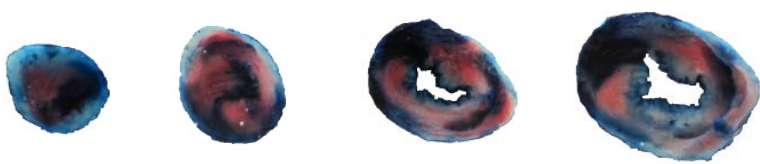

Pre-C
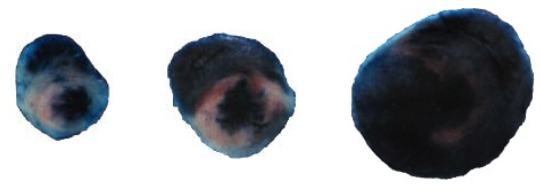

Per-C
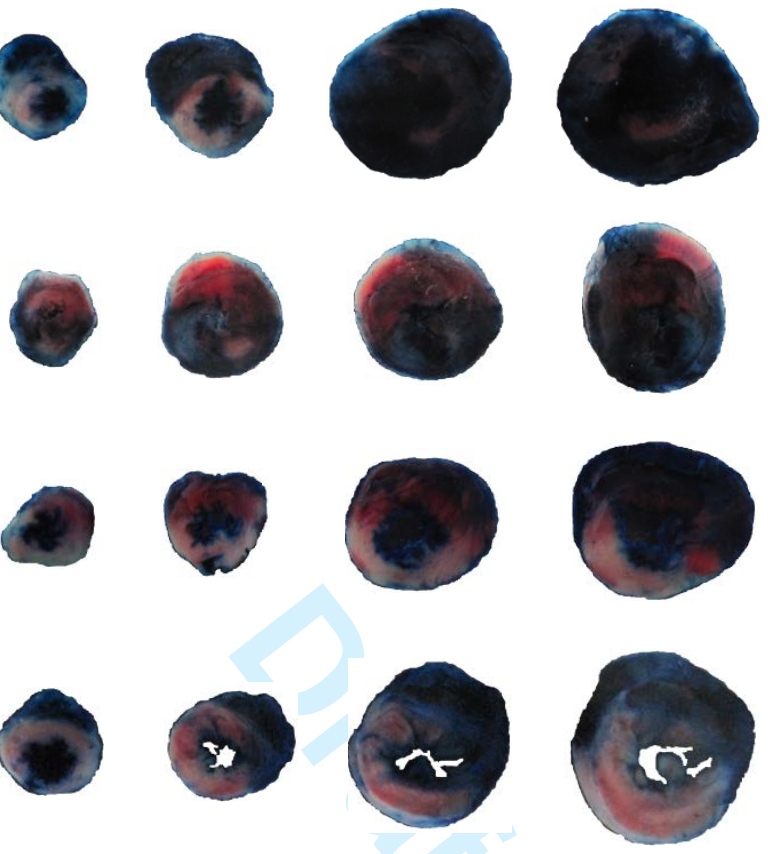

Gli

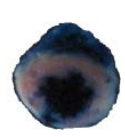

\section{(3)}
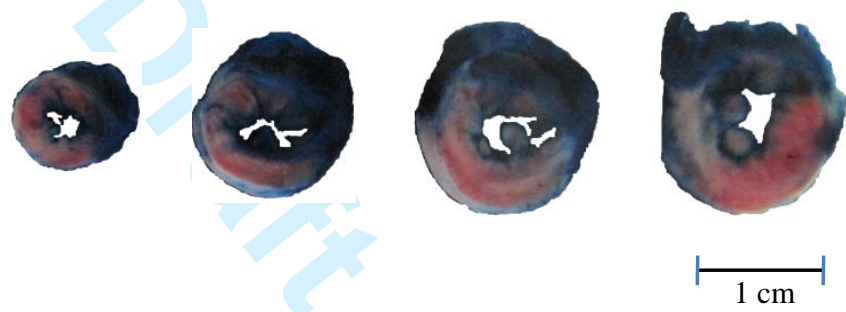

B

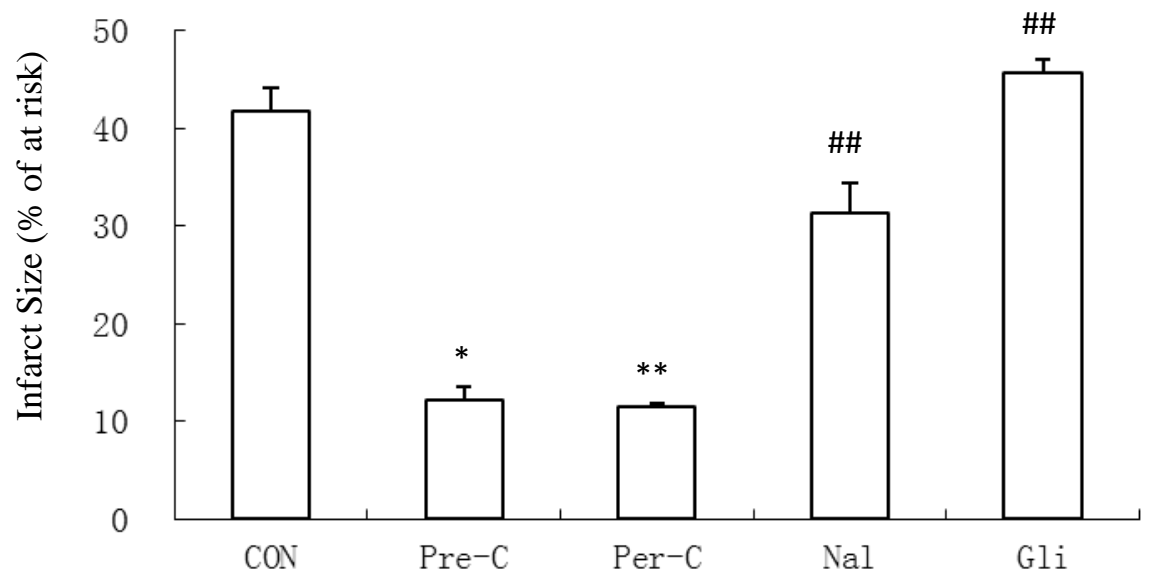




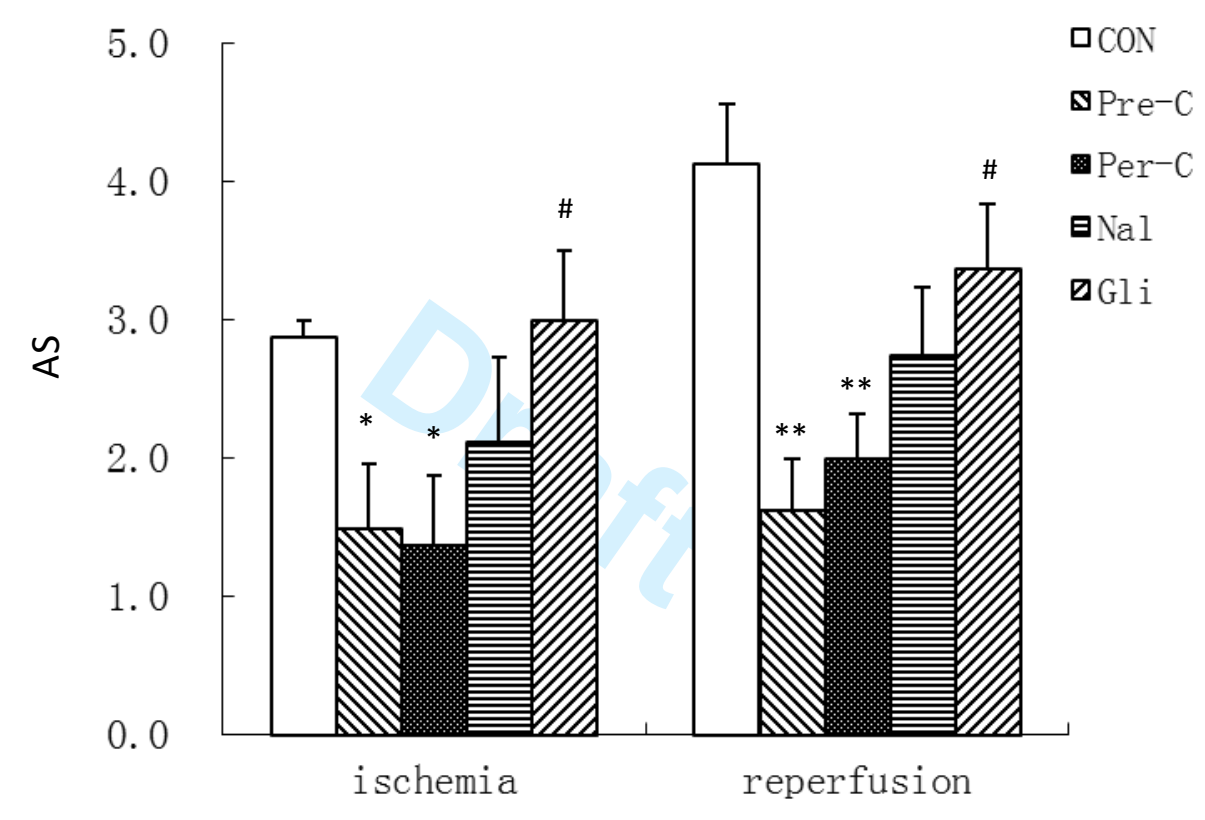

https://mc06.manuscriptcentral.com/cjpp-pubs 

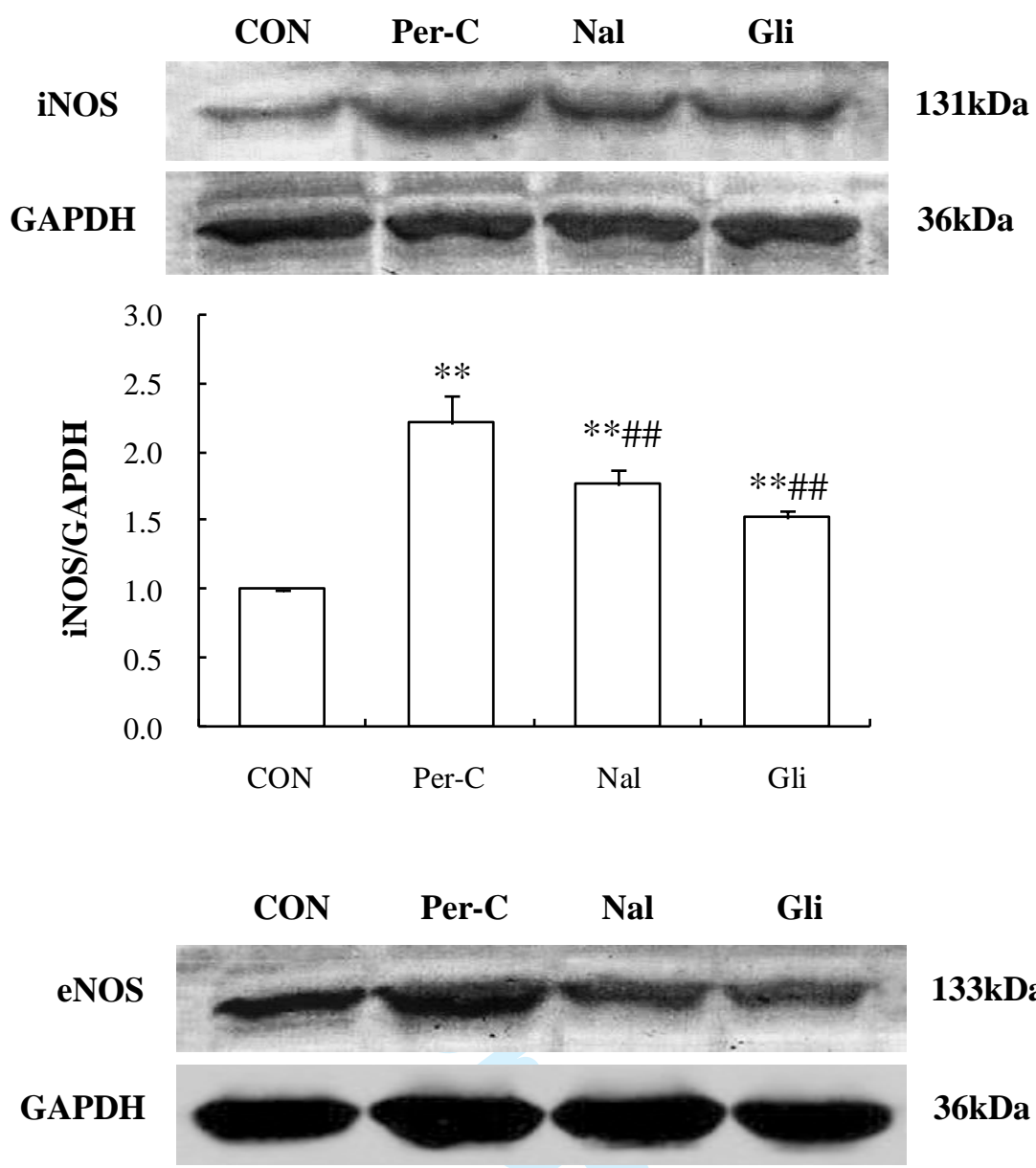

133kDa

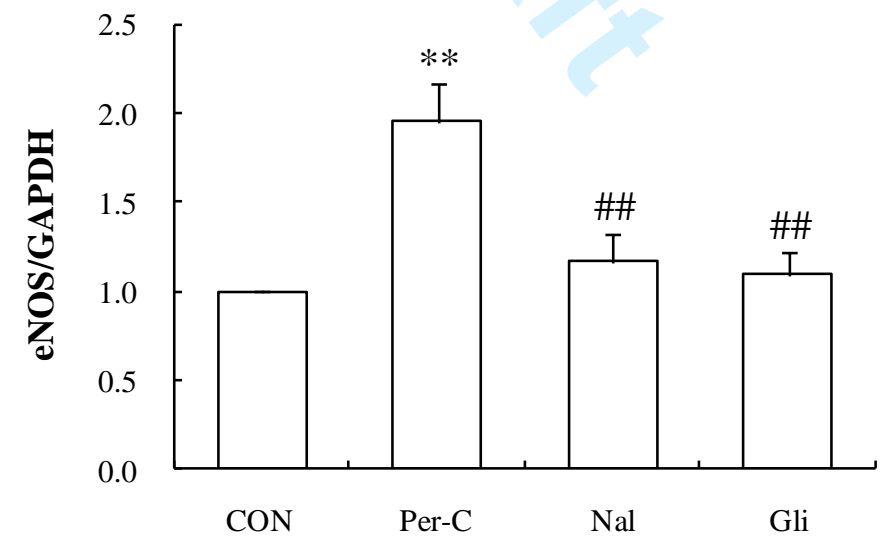

Figure 4 

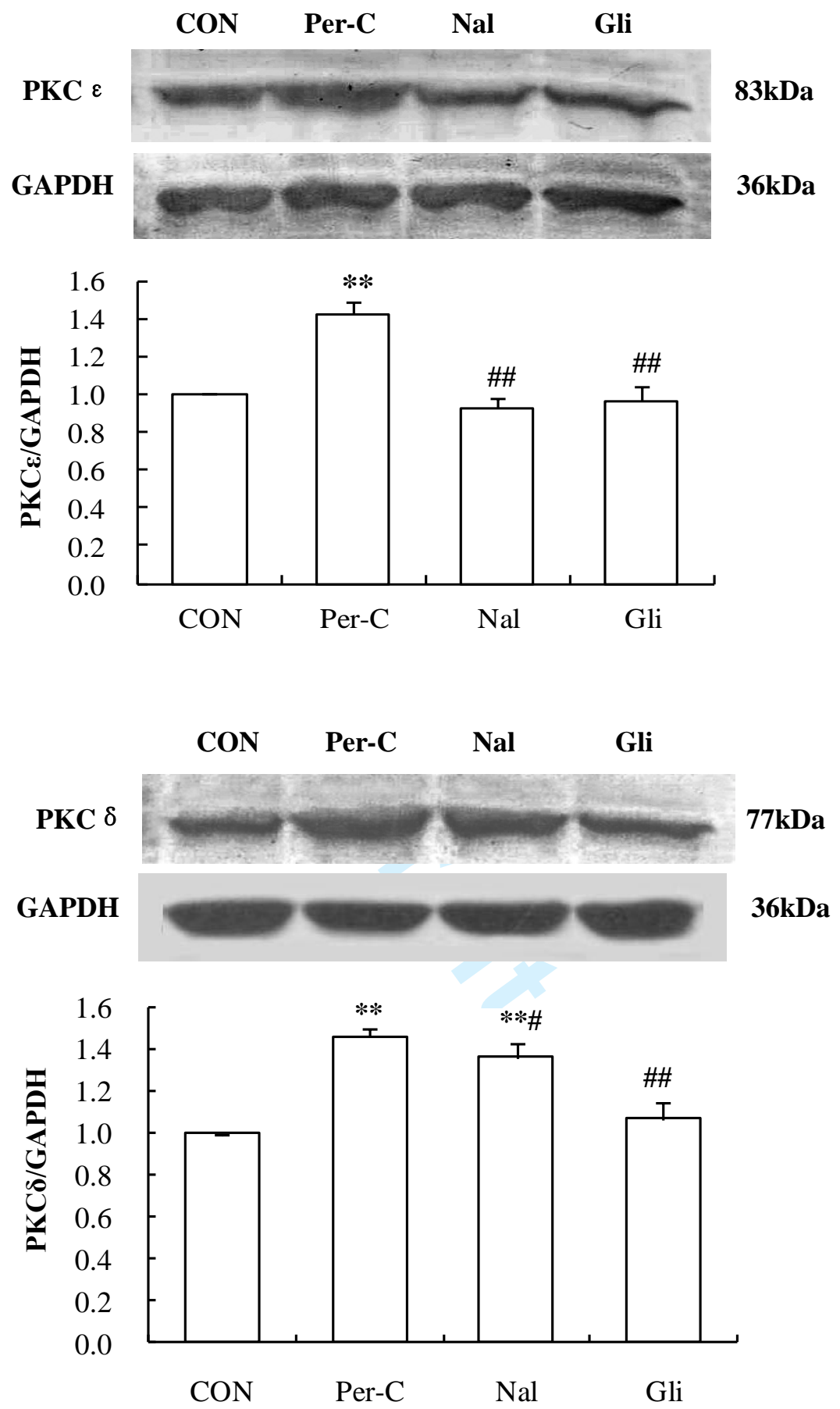

Figure 5 